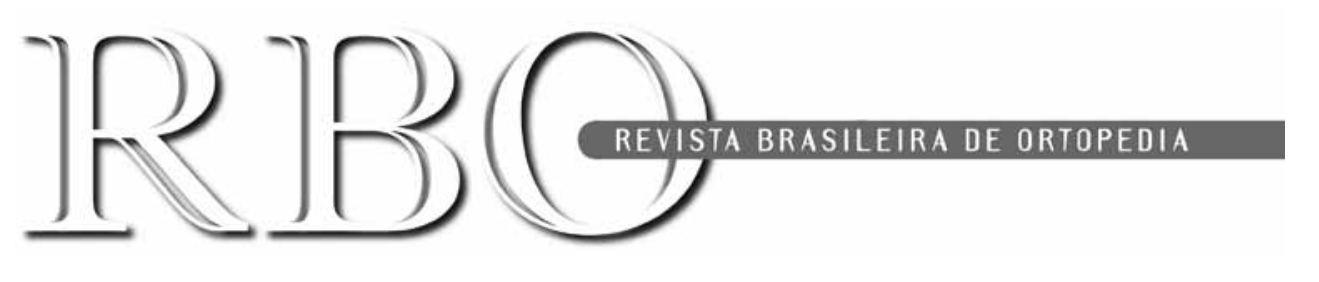

\title{
Editorial
}

\section{EDITING THE RBO}

Editing the RBO is an agreeable task but entails great responsibility, because each edition will be judged by more than 8,000 Brazilian orthopedists and by colleagues within the world of orthopedics through the electronic version, which has existed since 1993. This version, in English and in Portuguese, is available through the portal www.rbo.org.br; it disseminates our journal worldwide and allows Brazilian readers to access any study published over this period.

There are no set rules defining how to edit a medical journal; nonetheless, we have established some standards that we seek to maintain, so that the composition of the journal is harmonious.

We start the journal with updating articles. In the beginning, the editor would have to implore selected authors to produce such studies; with the passage of time, these requests started to be attended promptly and today they are offered by authors. These articles are on topics that we deem to be current and are written by authors with links to the subject. They are not screened by the editorial board but are discussed between the authors and the editor. These contributions provide a snapshot of a given subject and are of great value.

In the present issue, there is a novelty: 3D spectacles for an updating article on threedimensional anatomical studies of the knee. This technology opens great prospects for illustrations in future published papers.

The body of the journal is composed of original articles, which form the character and basic content of the publication. These are studies that initially were submitted to the editor and then to the editorial body. These texts are reviewed by at least two editors, who may be members of the editorial board or area editors suggested by the committees. Around $70 \%$ of the studies are returned to the authors after the editors' first analysis, for adjustments to be made.

The rejection rate among the original studies submitted is 18 to $20 \%$. Some are rejected by the editor because they are incompatible with the spirit of the RBO, while others (the majority) are 
rejected by the editors because they do not fulfill the requirements that are deemed necessary. In some cases, such rejections occur even after the corrections that are requested.

We have already received more than 700 studies over the last 32 months, i.e. the length of time for which the RBO's present editorial board has served. Some specialties have had greater production than others, but we have sought to maintain some degree of proportionality in the journal's composition. With regard to original studies, those on general topics and on trauma always have priority, followed by those from different specialties and finally by basic science texts and technical notes. The order of publication obeys firstly the composition described above and then the date of approval.

The number of articles on basic science is small, and these are published quickly. On the other hand, articles on the shoulder and knee are submitted more frequently and therefore wait longer to be published.

Case reports, which some journals reject, are considered welcome in the RBO. These are submitted to selection by the editorial body that is as rigorous as for original studies, and the rejection rate is maintained at around $20 \%$. We believe that case reports are of great importance for describing rare situations; they are very useful in clinical practice and even as a matter of professional defense. These are published at the end of the journal, and there are always three or four of them, in a sequence that takes into consideration only the date of approval.

Today, our menu is composed of around 20 items, among updating articles, original studies and case reports, and these are published in bimonthly issues.

At the next meeting of the editorial board and of the body of editors, we will be discussing whether we should increase the number of articles or the number of issues of the RBO.

There is also a third possibility that some international journals have practiced, which would be publish part of the material in conventional form and, in this publication, to cite studies that will only be available in electronic form, but with the same citation and indexation importance. We are going to sound out our grassroots in this respect.

One type of publication that we regard with great sympathy is supplements. These are collections of texts organized by committees and evaluated by the editor, with a specific editorial body, which we believe to be of great value for readers.

In the past, we have had supplements on pediatric orthopedics, orthopedic trauma, sports medicine, osteometabolic disease and the hip. We are awaiting others that are at the final stage of organization.

This is the way in which we edit the RBO, which with this issue, goes into its third year under the direction of the present group of editors, reviewers and editing technicians. 